\title{
Adequacies of lymphadenectomy range for gastric cancer according to the second and third/fourth Japanese gastric cancer treatment guidelines
}

\author{
Yo-Seok Cho ${ }^{1}$, Hyuk-Joon Lee ${ }^{1,2}$, Shin-Hoo Park', Tae-Han Kim ${ }^{1,3}$, Hwi Nyeong Choe ${ }^{4}$, Yun-Suhk Suh', Seong-Ho Kong ${ }^{1}$, \\ Han-Kwang Yang ${ }^{1,2}$ \\ 'Department of Surgery, Seoul National University College of Medicine, Seoul; \\ ${ }^{2}$ Cancer Research Institute, Seoul National University College of Medicine, Seoul; \\ ${ }^{3}$ Department of Surgery, Gyeongsang National University School of Medicine, Jinju; \\ ${ }^{4}$ Department of Nursing, Seoul National University Hospital, Seoul, Korea
}

Purpose: This study evaluated the adequacies of lymph node (LN) dissection according to the second version (determined by tumor location) or third/fourth version (determined by surgery extent) of the Japanese gastric cancer treatment guidelines.

Methods: Prospectively collected data of 3,948 gastric cancer patients who underwent gastrectomy were analyzed. The prevalence of LN metastasis and 5-year survival were analyzed according to tumor invasion depth and tumor location. In early gastric cancer (EGC), the frequency of LNs were evaluated. In advanced gastric cancer (AGC), the frequency of LN metastasis and the 5 -year survival rate of patients with positive LN were evaluated.

Results: For lower-third EGC, the positive rates for the \#1 and \#4sb were $0.93 \%$ and $0 \%$. For upper-third EGC, the positive rates for \#4d, \#5, $\# 6$, and \#11p were $0.3 \%, 0 \%, 0.76 \%$, and $1.22 \%$. For lower-third AGC, the positive rates for \#4sb and \#14v were 2.48\% and 7.64\%, and the 5 -year survival rates were $69.2 \%$ and $12.5 \%$, respectively. For upper-third AGC, the positive rates for \#5, \#6, and \#12a were $2.33 \%, 2.57 \%$, and $2.03 \%$, and the 5 -year survival rates were $21.8 \%, 64.3 \%$, and $0 \%$, respectively.

Conclusion: According to our analysis, in EGC, LN dissection in second edition seems more suitable, however LN dissection in \#11p would be mandatory in upper third EGC. In AGC, LN dissection in third/fourth edition seems more suitable in terms of frequency of LN metastasis and survival rate.

Keywords: Gastric neoplasms, Lymph node excision, Gastrectomy, Guideline

\section{INTRODUCTION}

Surgery is the main curative treatment for gastric cancer [1,2], and prognosis can be predicted by peri-gastric lymph node (LN) metastasis and depth of invasion as part of the TNM staging [3,4]. Previous studies have revealed that complications, survival, and re-

Received: Oct 11, 2017 Accepted: Nov 1, 2017

Correspondence to: Hyuk-Joon Lee

Department of Surgery and Cancer Research Institute, Seoul National University College of Medicine, 103 Daehak-ro, Jongno-gu, Seoul 03080, Korea

Tel: +82-2-2072-1957, Fax: +82-2-766-3975

E-mail: appe98@snu.ac.kr

Copyright (C) Korean Society of Surgical Oncology

This is an Open Access article distributed under the terms of the Creative Commons Attribution Non-Commercial License (http://creativecommons.org/licenses/by-nc/4.0) which permits unrestricted non-commercial use, distribution, and reproduction in any medium, provided the original work is properly cited. currence may vary according to the range of LN dissection [5-8]. For example, the Dutch trial revealed that $\mathrm{D} 2$ dissection decreased the rates of cancer-related mortality and local recurrence (vs. D1 dissection), but increased the rates of operative mortality and complications [5,6]. In contrast, the Japan Clinical Oncology Group trial compared D2 and para-aortic dissection (D3), and failed to detect differences in recurrence-free survival, although D3 dissection was associated with more complications, bleeding, and operative time [7]. Other studies have also indicated that more radical dissection is not beneficial for improving the prognosis of patients with gastric cancer $[8,9]$. Thus, it is important to determine the appropriate range for $\mathrm{LN}$ dissection in cases of gastric cancer.

The Japanese Gastric Cancer Association (JGCA) has proposed recommendation for LN dissection in cases of gastric cancer [1012], which generally involves D1 or D1+ dissection for early gastric cancer (EGC) and D2 dissection for advanced gastric cancer 
(AGC). However, the second and third/fourth editions of the JGCA treatment guidelines have minor differences, as the dissection range was determined using tumor location in the second edition, compared to gastrectomy extent in the third/fourth edition. Although a simple comparison is difficult, as the extent of gastrectomy depends on the tumor location, the frequencies of LN metastasis for specific tumor locations may help determine the appropriate extent of LN dissection [13]. Therefore, the present study aimed to evaluate the adequacies of $\mathrm{LN}$ dissection extent based on the second and third/fourth editions of the JGCA guidelines, using the rates of LN metastasis and survival for each positive LN station.

\section{METHODS}

This retrospective study examined prospectively collected data from 3,948 patients who underwent gastrectomy for gastric cancer at Seoul National University Hospital between January 2011 and December 2015. The study's protocol was approved by the Institutional Review Board (IRB no. 1707-068-869), and the data were collected from electronic medical records while protecting patient anonymity.

We followed American Joint Committee on Cancer seventh edition for gastric cancer staging. The grouping of LNs was performed by the attending surgeon immediately after the gastrectomy, and the LN stations were classified according to the JGCA system [14]. Tumor location was defined as the upper-third, middle-third, or lower-third of the stomach; cases that involved the entire stomach were excluded. Surgery after endoscopic submucosal dissection was performed for cases with a possibility of LN metastasis [15], and invasion to other organs was treated in an attempt to improve the patient's prognosis. The tumor's depth was classified as EGC or AGC, based on the pathological results.

To compare the second and third/fourth editions of the JGCA guidelines, upper-third lesions were assumed to have been treated using total gastrectomy, while middle-third and lower-third lesions were assumed to have been treated using distal gastrectomy. Table 1 summarizes the LN dissection extents from the two guidelines, based on the assumption that $\mathrm{D} 1+\beta$ (second edition) or $\mathrm{D} 1+$ (third/fourth edition) were the standard extents for EGC, with D2 being the standard extent for AGC. The patients' data were also examined to determine the frequency of LN metastasis in EGC cases, as well as survival and specific LN metastases in AGC cases.

There was no difference between the second and third/fourth versions for middle-third gastric cancer. Thus, we only investigated gastric cancers in the lower-third and upper-third. For each station, the frequency of LN metastasis and survival rate were evaluated, with frequency being defined as the percentage of positive LNs among the resected LNs, which is a useful prognostic factor [4]. Stations were considered mandatory for dissection (1) if the frequency of $L N$ metastasis was $\geq 1 \%$ in EGC cases or (2) patients with positive LNs and AGC had a 5 -year survival rate of $\geq 20 \%$. In some papers investigating the frequency of LN metastasis in EGC, the incidence of perigastric lymph nodes was reported to be $1.2 \%-7 \%$, suggesting that more than $1 \%$ of $\mathrm{LN}$ metastasis woule be suitable for mandatory dissection [16-19]. Also in our hospital data, 5-year survival after curative resection of gastric cancer showed that stage IIIa was $53.9 \%$, IIIb was $36.5 \%$, and IV was $23.9 \%$. So, we think that 5 -year survival showed over 20\% than the dissection would be meaningful [1].

Data were analyzed using IBM SPSS software ver. 21 (IBM Corp., Armonk, NY, USA) and Excel software (version 2013). Data were reported as number (\%) and compared using frequency analysis. Survival outcomes were compared using the Kaplan-Meier method.

\section{RESULTS}

\section{Demographic characteristics}

Table 2 shows the patients' demographic characteristics. The mean

Table 1. Comparing lymph node dissection between the second and third/fourth editions according to tumor location

\begin{tabular}{|c|c|c|}
\hline & Second edition (D1+B for EGC/D2 for AGC) & Third/Fourth edition (D1+ for EGC/D2 for AGC) \\
\hline \multicolumn{3}{|l|}{ EGC } \\
\hline Lower third & $3,4 d, 5,6,7,8 a, 9$ & $1^{\text {a) }}, 3,4 s b^{a}, 4 d, 5,6,7,8 a, 9$ \\
\hline Middle third & $1,3,4 s b, 4 d, 5,6,7,8 a, 9$ & $1,3,4 s b, 4 d, 5,6,7,8 a, 9$ \\
\hline Upper third & $1,2,3,4 s a, 4 s b, 7,8 a, 9$ & $1,2,3,4 s a, 4 s b, 4 d^{a}, 5^{a)}, 6^{a}, 7,8 a, 9,11 p^{a)}$ \\
\hline \multicolumn{3}{|l|}{$A G C$} \\
\hline Lower third & $1,3,4 d, 5,6,7,8 a, 9,11 p, 12 a, 14 v^{a)}$ & $1,3,4 s b^{a}, 4 d, 5,6,7,8 a, 9,11 p, 12 a$ \\
\hline Middle third & $1,3,4 s b, 4 d, 5,6,7,8 a, 9,11 p, 12 a$ & $1,3,4 s b, 4 d, 5,6,7,8 a, 9,11 p, 12 a$ \\
\hline Upper third & $1,2,3,4 s a, 4 s b, 4 d, 7,8 a, 9,10,11 p, 11 d$ & $1,2,3,4 s a, 4 s b, 4 d, 5^{a)}, 6^{a)}, 7,8 a, 9,10,11 p, 11 d, 12 a^{a}$ \\
\hline
\end{tabular}

EGC, early gastric cancer; AGC, advanced gastric cancer.

a) Differences between the second and third editions. 
Table 2. The patients' demographic characteristics

\begin{tabular}{|c|c|}
\hline Characteristic & Value \\
\hline Age (yr) & $60.39 \pm 11.95$ \\
\hline \multicolumn{2}{|l|}{ Sex } \\
\hline Male & $2,538(64.3)$ \\
\hline Female & $1,410(35.7)$ \\
\hline \multicolumn{2}{|l|}{ Tstage } \\
\hline T1 & $2,488(63.0)$ \\
\hline $\mathrm{T} 2$ & $489(12.4)$ \\
\hline T3 & $525(13.3)$ \\
\hline T4 & $446(11.3)$ \\
\hline \multicolumn{2}{|l|}{ N stage } \\
\hline NO & $2,769(70.1)$ \\
\hline N1 & $420(10.6)$ \\
\hline N2 & $363(9.2)$ \\
\hline N3 & $396(10.0)$ \\
\hline \multicolumn{2}{|l|}{ Tumor location } \\
\hline Lower & $2,165(54.8)$ \\
\hline Middle & $1,093(27.7)$ \\
\hline Upper & $690(17.5)$ \\
\hline \multicolumn{2}{|l|}{ Extent of resection } \\
\hline Distal gastrectomy & $2,314(58.6)$ \\
\hline Total gastrectomy & $757(19.2)$ \\
\hline Proximal gastrectomy & $113(2.9)$ \\
\hline Pylorus-preserving gastrectomy & 764 (19.4) \\
\hline \multicolumn{2}{|l|}{ Approach } \\
\hline Open & $1,457(36.9)$ \\
\hline Laparoscopy & $2,268(57.4)$ \\
\hline Robot & $223(5.6)$ \\
\hline
\end{tabular}

Values are mean \pm standard deviation or number $(\%)$.

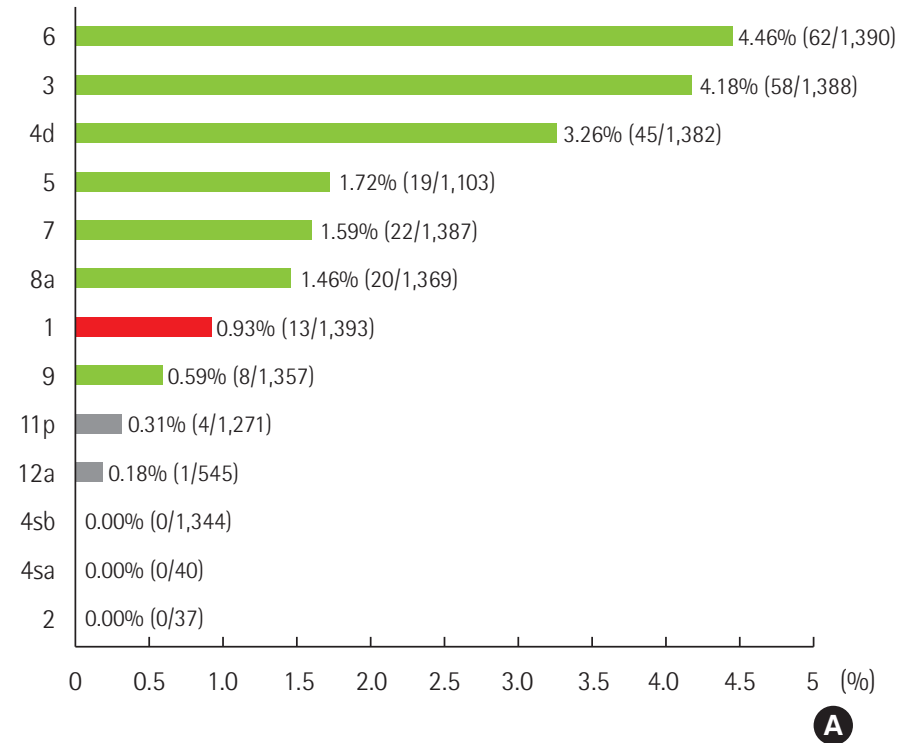

age was $60.39 \pm 11.95$ years. Most patients were men $(64.3 \%$, $\mathrm{n}=2,538)$, had EGC $(63.0 \%, \mathrm{n}=2,488)$, and did not have LN metastasis $(70.1 \%, \mathrm{n}=2,769)$. The cases with $\mathrm{LN}$ metastasis involved $\mathrm{N} 1$ metastasis $(10.6 \%, \mathrm{n}=420), \mathrm{N} 2$ metastasis $(9.2 \%, \mathrm{n}=363)$, and $\mathrm{N} 3$ metastasis $(10.0 \%, \mathrm{n}=396)$. The tumor locations were upper-third $(17.5 \%, \mathrm{n}=690)$, middle-third $(27.7 \%, \mathrm{n}=1,093)$, and lower-third $(54.8 \%, \mathrm{n}=2,165)$. Most patients underwent distal gastrectomy $(58.6 \%, \mathrm{n}=2,314)$ or total gastrectomy $(19.2 \%, \mathrm{n}=757)$. Smaller proportions of patients underwent proximal gastrectomy $(2.9 \%, \mathrm{n}=113)$ or pylorus-preserving gastrectomy $(19.4 \%, \mathrm{n}=$ 764).

\section{Adequacy of LN dissection for EGC}

Fig. 1 shows the frequencies of LN metastasis from lower-third or upper-third EGC. For lower-third EGC, stations 1 and 4sb were added to the third/fourth edition and had metastasis rates of $0.93 \%$ and $0 \%$, respectively. For upper-third EGC, stations 4d, 5, 6, and $11 \mathrm{p}$ were added to the third/fourth edition as D1+LN. Only $11 \mathrm{p}$ had a metastasis rate of $>1 \%(1.22 \%, 4 / 328)$, with lower rates for $4 \mathrm{~d}(0.3 \%, 1 / 332), 5(0 \%, 0 / 226)$, and $6(0.76 \%, 2 / 262)$.

\section{Adequacy of LN dissection for AGC}

Fig. 2 shows the frequencies of $\mathrm{LN}$ metastasis and survival for lower-third or upper-third AGC. For lower-third AGC, station 4sb was added and $14 \mathrm{v}$ was removed from the third/fourth edition, and had metastasis rates of $2.48 \%(18 / 726)$ and $7.64 \%(12 / 157)$, respectively. Patients with $4 \mathrm{sb}$ metastasis had a 5 -year survival rate of $69.2 \%$,

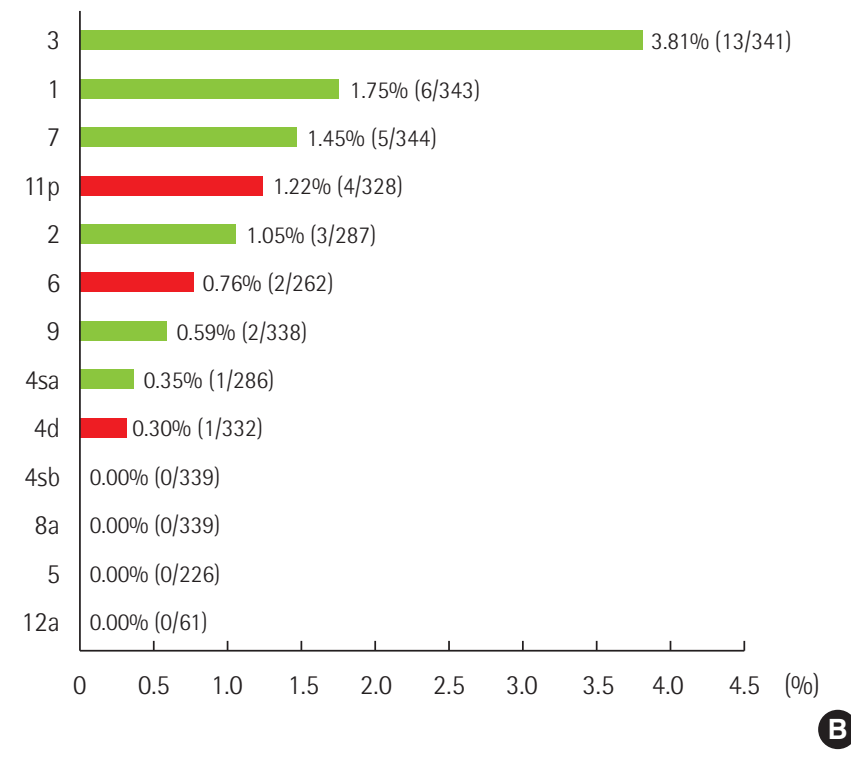

Fig. 1. Frequency of lymph node (LN) metastasis in early gastric cancer (EGC). (A) LN metastasis (\%) in the lower third EGC. (B) LN metastasis (\%) in the upper third EGC. Green bar means LN station within D1+ in the second and third/fourth editions. Red bar means LN station beyond D1+ in the second edition, but within D1+ in the third/fourth edition. Grey bar means LN station beyond D1+ in the second and third/fourth editions. 

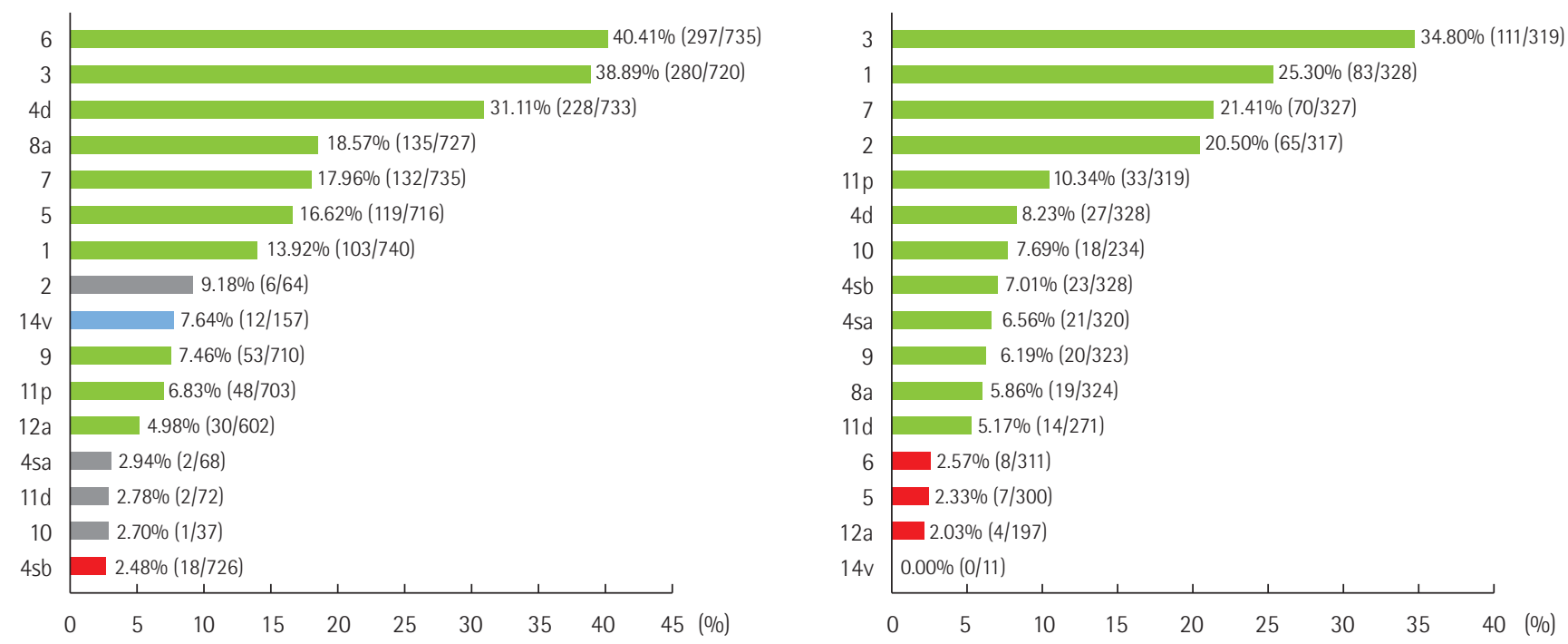

A
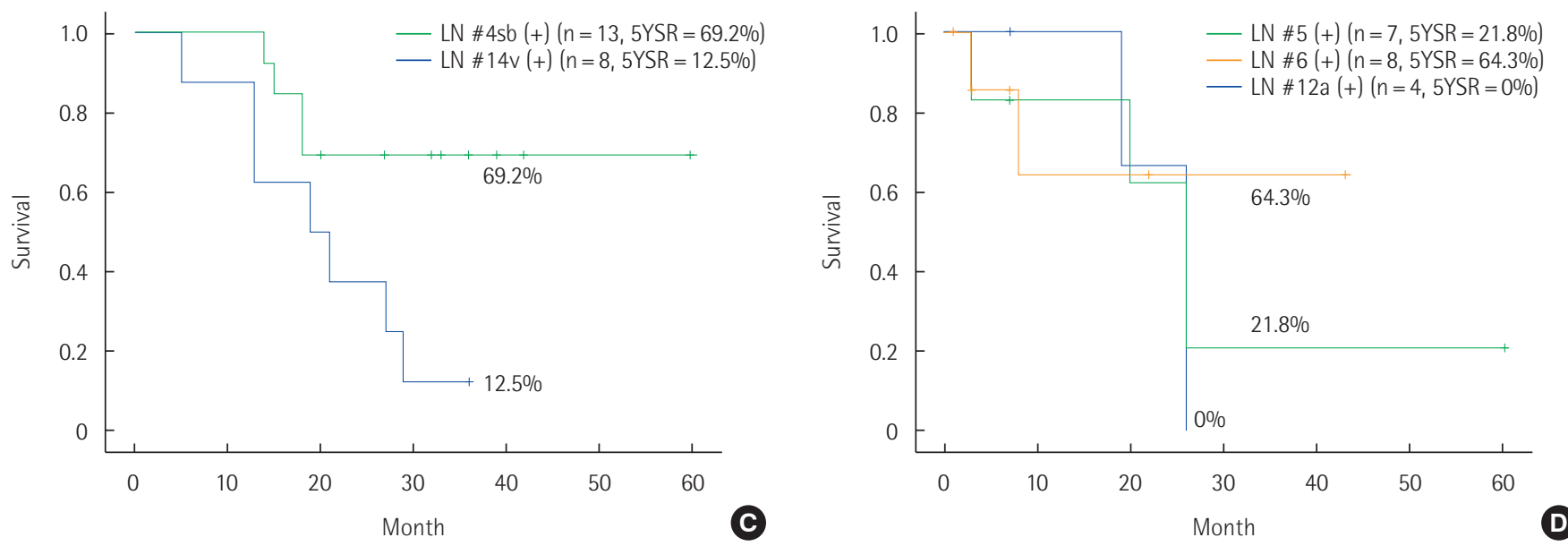

Fig. 2. Frequency of lymph node (LN) metastasis in advanced gastric cancer (AGC) and survival according to specific metastases. (A) LN metastasis (\%) in the lower third AGC. (B) LN metastasis (\%) in the upper third AGC. (C) Survival curve in the lower third AGC. (D) Survival curve in the upper third AGC. Green bar means LN station within D2 in the second and third/fourth editions. Red bar means LN station beyond D2 in the second edition, but within D2 in the third/fourth edition. Grey bar means LN station beyond D2 in the second and third/fourth editions. Blue bar means LN station within D2 in the second edition, but beyond D2 in the third/fourth edition. 5YSR, 5-year survival rates.

while patients with $14 \mathrm{v}$ metastasis had a 5-year survival rate of just 12.5\%. For upper-third AGC, stations 5, 6, and 12a were added to the third/fourth edition, and had metastasis rates of $2.33 \%, 2.57 \%$, and $2.03 \%$, respectively. Patients with station 5 metastasis had a 5 -year survival rate of $21.8 \%$. Among the eight patients with station 6 metastasis, the 5 -year survival rate was $64.3 \%$. All four patients who had station 12 a metastasis died within 3 years.

\section{DISCUSSION}

Gastric cancer frequently leads to LN metastasis, which is the most important prognostic factor for gastric cancer $[4,20]$, and it is im- portant to understand the adequacy of LN dissections. Our center and many other Korean hospitals follow the JGCA recommendations for treating gastric cancer. Thus, it was important for us to examine the changes in the second and third/fourth editions of the JGCA guidelines, and to question whether location-based dissection would be more appropriate.

For lower-third EGC, dissection of stations 1 and 4 sb is not mandatory, because the metastasis frequencies were $<1 \%$. However, for AGC, the metastasis rates were $>1 \%$ for stations $14 \mathrm{v}$ and $4 \mathrm{sb}$, which indicates that their dissection should be considered mandatory in terms of frequency. Furthermore, $11 \mathrm{p}$ dissection for upper-third EGC was added in the third / fourth edition, and had 
a metastasis rate of $>1 \%$, which indicates that it should be considered mandatory. Nevertheless, the 4 patients with $11 \mathrm{p}$ metastasis from upper-third EGC survived throughout the follow-up period. Stations 5 and 6 had metastasis rates of $<1 \%$ and should not be considered mandatory for dissection.

We considered the survival of all patients with lower-third AGC because they had a metastasis rate of $<1 \%$. In that analysis, the 5-year survival rates were $69.2 \%$ for $4 \mathrm{sb}$ and $12.5 \%$ for $14 \mathrm{v}$ (most patients with $14 \mathrm{v}$ metastasis died, and the one surviving patient did not complete the 5-year follow-up). These results indicate that 4sb dissection should be considered mandatory for lower-third AGC, although $14 \mathrm{v}$ dissection is less important, which favors the third/fourth edition of the JGCA guidelines. Interestingly, the four patients with $11 \mathrm{p}$ metastasis from upper-third EGC survived, which suggests that $11 \mathrm{p}$ dissection should be considered mandatory based on the third/fourth edition of the JGCA guidelines. However, among AGC cases, the 5-year survival rates were $21.8 \%$ for station $5,64.3 \%$ for station 6 , and $0 \%$ for station $12 \mathrm{a}$, which suggests that 12 a dissection is not mandatory based on the second edition, despite the relatively high metastasis rate. This may be because all four patients with 12a metastasis died because of recurrence within 3 years after surgery. A low 5 -year survival rate was observed for patients with station 5 metastasis, although one of the five patients survived to 5 years without recurrence, which suggests that this is a mandatory dissection.

The present study has several limitations. There are too small patients in each survival analyses ranging 4 to 13 patients. Although this data showed the trend of survival rate but it does not have statistical significance. To see the survival, multivariable factors should be considered but excluding some specific lymph nodes, we didn't analyze all the factors influencing the survival could not be investigated. And about 20\% of the patients were underwent limited gastrectomy with limited LN dissection such as proximal gastrectomy or pylorus preserving gastrectomy, and it that might affect the frequency of LN mestastasis in EGC.

In conclusion, we assessed the adequacies of $\mathrm{LN}$ dissection based on the JGCA guidelines, which had several changes between the second and third/fourth editions, especially for AGC. In cases of lower-third EGC, dissection of stations 1 and 4sb is not mandatory, because of their low metastasis rates. However, in cases of lower-third AGC, it is reasonable to consider 4sb mandatory for dissection and to consider omitting $14 \mathrm{v}$ dissection. In cases of upper-third EGC, dissection of stations 4d, 5, and 6 does not appear to be mandatory, which favors the second edition, although $11 \mathrm{p}$ dissection is mandatory, which favors the third/fourth edition. In cases of upper-third AGC, dissection of 12a does not appear to be mandatory, while dissection of stations 5 and 6 is mandatory, which favors the third/fourth edition.

\section{CONFLICT OF INTEREST}

No potential conflict of interest relevant to this article was reported.

\section{REFERENCES}

1. Lee HJ, Yang HK, Ahn YO. Gastric cancer in Korea. Gastric Cancer 2002;5:177-82.

2. Ahn HS, Lee HJ, Hahn S, Kim WH, Lee KU, Sano T, et al. Evaluation of the seventh American Joint Committee on Cancer/International Union Against Cancer Classification of gastric adenocarcinoma in comparison with the sixth classification. Cancer 2010; 116:5592-8.

3. Maruyama K, Gunven P, Okabayashi K, Sasako M, Kinoshita T. Lymph node metastases of gastric cancer. General pattern in 1931 patients. Ann Surg 1989;210:596-602.

4. Kim JP, Lee JH, Kim SJ, Yu HJ, Yang HK. Clinicopathologic characteristics and prognostic factors in 10,783 patients with gastric cancer. Gastric Cancer 1998;1:125-33.

5. Bonenkamp JJ, Hermans J, Sasako M, van de Velde CJ, Welvaart K, Songun I, et al. Extended lymph-node dissection for gastric cancer. N Engl J Med 1999;340:908-14.

6. Songun I, Putter H, Kranenbarg EM, Sasako M, van de Velde CJ. Surgical treatment of gastric cancer: 15-year follow-up results of the randomised nationwide Dutch D1D2 trial. Lancet Oncol 2010; 11:439-49.

7. Sasako M, Sano T, Yamamoto S, Kurokawa Y, Nashimoto A, Kurita A, et al. D2 lymphadenectomy alone or with para-aortic nodal dissection for gastric cancer. N Engl J Med 2008;359:453-62.

8. Tokunaga M, Ohyama S, Hiki N, Fukunaga T, Inoue H, Yamada K, et al. Therapeutic value of lymph node dissection in advanced gastric cancer with macroscopic duodenum invasion: is the posterior pancreatic head lymph node dissection beneficial? Ann Surg Oncol 2009;16:1241-6.

9. Sano T, Sasako M, Mizusawa J, Yamamoto S, Katai H, Yoshikawa T, et al. Randomized controlled trial to evaluate splenectomy in total gastrectomy for proximal gastric carcinoma. Ann Surg 2017;265: 277-83.

10. Nakajima T. Gastric cancer treatment guidelines in Japan. Gastric Cancer 2002;5:1-5.

11. Japanese Gastric Cancer Association. Japanese gastric cancer treatment guidelines 2010 (ver. 3). Gastric Cancer 2011;14:113-23.

12. Japanese Gastric Cancer Association. Japanese gastric cancer treatment guidelines 2014 (ver. 4). Gastric Cancer 2017;20:1-19.

13. Kampschoer GH, Maruyama K, van de Velde CJ, Sasako M, 
Kinoshita T, Okabayashi K. Computer analysis in making preoperative decisions: a rational approach to lymph node dissection in gastric cancer patients. Br J Surg 1989;76:905-8.

14. Japanese Gastric Cancer Association. Japanese classification of gastric carcinoma: 3rd English edition. Gastric Cancer 2011;14:101-12.

15. Hirasawa T, Gotoda T, Miyata S, Kato Y, Shimoda T, Taniguchi H, et al. Incidence of lymph node metastasis and the feasibility of endoscopic resection for undifferentiated-type early gastric cancer. Gastric Cancer 2009;12:148-52.

16. Ooki A, Yamashita K, Kikuchi S, Sakuramoto S, Katada N, Hutawatari $\mathrm{N}$, et al. Clinical significance of total gastrectomy for proximal gastric cancer. Anticancer Res 2008;28:2875-83.
17. Kunisaki C, Shimada H, Nomura M, Akiyama H. Appropriate lymph node dissection for early gastric cancer based on lymph node metastases. Surgery 2001;129:153-7.

18. Ren G, Cai R, Zhang WJ, Ou JM, Jin YN, Li WH. Prediction of risk factors for lymph node metastasis in early gastric cancer. World J Gastroenterol 2013;19:3096-107.

19. Han KB, Jang YJ, Kim JH, Park SS, Park SH, Kim SJ, et al. Clinical significance of the pattern of lymph node metastasis depending on the location of gastric cancer. J Gastric Cancer 2011;11:86-93.

20. de Manzoni G, Verlato G, Guglielmi A, Laterza E, Genna M, Cordiano C. Prognostic significance of lymph node dissection in gastric cancer. Br J Surg 1996;83:1604-7. 